\section{Trombose arterial em leucemia promielocítica aguda}

\section{Arterial thrombosis in acute promyelocytic leukemia}

Sonia Regina Iantas ${ }^{1}$

Ricardo A. Fock ${ }^{2}$

${ }^{1}$ Centro Médico Hospitalar Pitangueiras/Sobam, Jundiaí, SP

${ }^{2}$ Laboratório de Análises Clínicas Anchieta/Grupo Unilab, Jundiaí, SP

\section{Senhor Editor}

Paciente do sexo masculino, de 56 anos, procurou o pronto-socorro com história compatível com claudicação intermitente em membro inferior direito há aproximadamente 10 dias. Não havia antecedentes prévios de doença arterial e não era tabagista. Fez uso de antiinflamatório não esteroidal, porém apresentando pouca melhora. Ao exame físico apresentava pé direito frio e cianótico e redução importante do pulso femoral. Os exames laboratoriais apresentaram concentração de hemoglobina de 14,0 g/dL, discreta leucocitose $12.2 \times 10^{9} / \mathrm{L}$, sem desvio à esquerda, com um TTPa $=31,1$ s e $\mathrm{R}=0,82$.

Diagnosticada insuficiência arterial aguda, foi imediatamente submetido à tromboembolectomia com a retirada de um trombo branco. Seu pós-operatório na UTI transcorreu sem intercorrências e o paciente foi mantido sob heparinização plena. No segundo pós-operatório, o paciente já não referia mais dor e sem evidências de novo processo trombótico. Entretanto, chamou a atenção do intensivista o seguinte hemograma: concentração de hemoglobina igual a 12.1g/dL, Leucócitos $1.7 \times 10^{9} / \mathrm{L}$, com valores absolutos de granulócitos igual a $0.35 \times 10^{9} / \mathrm{L}$ e número total de plaquetas igual a $63 \times 10^{9} / \mathrm{L}$. Um novo hemograma foi solicitado após 24 horas e apresentava características semelhantes, sem mudança do quadro.

Solicitada avaliação hematológica, o mielograma mostrou: medula óssea hipercelular à custa da série granulocítica, sendo $67 \%$ promielócitos e $17 \%$ mieloblastos, com presença de bastonetes de Aüer e reação de peroxidase fortemente positiva. A imunofenotipagem mostrou marcação para os antígenos de superfície: CD117+, CD33+, CD13+ e MPO+, e a citogenética revelou: 46,XY, t(15;17)(q22;q21) que confirmaram o diagnóstico de leucemia promielocítica aguda (LPA).

A LPA corresponde de $10 \%$ a $15 \%$ das leucemias agudas, sendo que $90 \%$ dos casos estão associados a translocação $(15,17)$ (q22;q21); clinicamente distingue-se pela elevada freqüência com que se acompanha de coagulação intravascular disseminada (CIVD) e pela boa resposta ao tratamento com ácido alfa-transretinóico (ATRA). ${ }^{1} \mathrm{O}$ desenvolvimento de trombose arterial é pouco freqüente, sendo encontrado na literatura poucos casos com essa característica ini- cial; estima-se que 9.6\% dos casos de $\mathrm{LPA}^{2}$ podem apresentar um perfil trombótico, entretanto complicações hemorrágicas são mais comuns. A fisiopatotologia da trombose arterial permanece pouco clara, na LPA, mas acredita-se que uma menor expressão de trombomodulina e moléculas de adesão possam estar envolvidas, propiciando o desenvolvimento de uma endotélio pró-coagulante. ${ }^{1,3,4}$

O tratamento da LPA foi iniciado com ácido alfa-transretinóico (ATRA) na dose de $45 \mathrm{mg} / \mathrm{m}^{2}$ V.O. e quimioterapia de indução com idarrubicina. O ATRA, além de agir como um agente de diferenciação celular, diminui a expresssão do fator tecidual e da cisteína proteinase câncer procoagulante e aumenta a expressão de trombomodulina, os quais são fatores importantes na contenção da CIVD., ${ }^{1,3,5}$

Durante o tratamento, o paciente foi mantido sob terapêutica anticoagulante; entretanto, 48 horas depois do início do tratamento desenvolveu quadro hemorrágico pela ferida cirúrgica, além de sangramento gengival e equimoses pelo corpo. A avaliação laboratorial mostrou: $\mathrm{TP}=24,3 \mathrm{~s}$; $\mathrm{RNI}=1,83$; $\mathrm{TTPa}=80,3 \mathrm{~s} \mathrm{R}=2,13$ e fibrinogênio $=156 \mathrm{mg} / \mathrm{dL}$, levando ao diagnóstico de CIVD. O paciente foi tratado com PFC (plasma fresco congelado) e heparina em baixa dose, com controle do quadro, e evoluiu satisfatoriamente, recebendo alta 25 dias após o inicio do tratamento, continuando o tratamento ambulatorialmente.

Resultados atuais mostram que o paciente encontra-se em remissão completa e sua nova avaliação citogenética mostrou negativação da $\mathrm{t}(15 ; 17)$.

\section{Abstract}

Acute promyeloclocytic leukemia can present coagulopathies which are frequently very serious due to hemorrhagic conditions. Treatment using anthracyclines and retinoids provide a good response. The development of arterial thrombosis is uncommon. In this work a 56-year-old male patient with acute arterial insufficiency was evaluated. This patient was immediately submitted to thromboembolectomy with the removal of a white thrombus. Postoperative tests showed acute promyelocytic leukemia with transposition $(15 ; 17)$ Treatment with ATRA and Idarubicin chemotherapy was initiated with the patients's response being satisfactory. Currently, the patient is incomplete remission and a recent cytogenetics test does not show the t(15;17). Rev. bras. hematol. hemoter. 2007; 29(4):418-419.

Key words: Acute promyelocytic leukemia; arterial thrombosis; treatment; citogenetic remission.

\section{Referências Bibliográficas}

1. Sharma JB, Gupta N, Vimala N, Anand M, Deka D, Mittal S. Acute promyelocytic leukemia: an unusual cause of fatal secondary postpartum hemorrhage. Arch Gynecol Obstet. 2006; 273(5):310-1.

2. De Stefano V, Sora F, Rossi E, Chiusolo P, Laurenti L, Fianchi L et al. The risk of thrombosis in patients with acute leukemia: occurrence of thrombosis at diagnosis and during treatment. J Thromb Haemost. 2005;3(9):1985-92. 
3. Kalk E, Goede A, Rose P. Acute arterial thrombosis in acute promyelocytic leukaemia. Clin Lab Haematol. 2003;25(4):267-70.

4. Barbui T, Falanga A. Disseminated intravascular coagulation in acute leukemia. Semin Thromb Hemost. 2001;27(6):593-604.

5. Gupta V, Yi QL, Brandwein J, Lipton JH, Messner HA, Schuh AC et al. Role of all-trans-retinoic acid (ATRA) in the consolidation therapy of acute promyelocytic leukaemia (APL). Leuk Res. 2005; 29(1):113-4.

Avaliação: Editor e dois revisores externos

Conflito de interesse: não declarado

Recebido: 26/05/2006

Aceito após modificações: 23/03/2007

Correspondência: Ricardo Ambrósio Fock

Rua Anchieta, 341

13201-804 - Jundiaí, SP

E-mail:ricfock@hotmail.com

\section{O fator estimulador de colônias granulocitárias (G-CSF) para isquemia cerebral. Uma nova aplicação terapêutica?}

\section{Granulocyte colony-stimulating factor (G-CSF) for ischemic stroke. A new therapeutic modality?}

\author{
Angelo Luiz Maset ${ }^{1}$ \\ Kleber P. Duarte ${ }^{2}$ \\ Oswaldo Tadeu Greco ${ }^{3}$
}

${ }^{1}$ Neurocirurgião - Associação Portuguesa de Beneficência de S.J. Rio Preto-SP; InNeuro - Instituto de Neurocirurgia e Neurociências do Oeste Paulista

${ }^{2}$ Neurocirurgião - Associação Portuguesa de Beneficência de S.J. Rio Preto-SP

${ }^{3}$ Cardiologista. Instituto de Moléstias Cardiovasculares de S.J. Rio Preto-SP

\section{Senhor Editor:}

O G-CSF é uma glicoproteína que foi descrita há mais de vinte anos, possui aprovação do FDA e é comumente utilizada para tratar neutropenia, ${ }^{1}$ ou para transplantes de medula óssea. ${ }^{2,3}$ Incluem-se como fontes de G-CSF os monócitos, células mesoteliais, fibroblastos e células endoteliais. O G-CSF estimula o crescimento de precursores de neutrófilos e regula crucialmente a sobrevivência de neu- trófilos maduros, pós-mitóticos, através da inibição da apoptose..$^{2,3,4}$ Desde o início desta década, vários pesquisadores têm demonstrado a surpreendente atividade do G-CSF no sistema nervoso central (SNC) ${ }^{5-8}$ A administração de GCSF mobiliza células-tronco e progenitoras da medula óssea para o sangue periférico, que por sua vez atravessam a barreira hemato-encefálica (BHE) e se dirigem à área acometida do cérebro. O G-CSF possui claramente um efeito neuroprotetor aparentemente multimodal, incluindo-se a atividade antiapoptóptica em neurônios, ${ }^{5,6}$ regeneração de vascularização, ${ }^{8}$ efeito antiinflamatório ${ }^{9}$ e estimulação de neurogênese endógena. ${ }^{10}$

Estes estudos promissores em animais deram o embasamento necessário para as primeiras utilizações um humanos. Ickenstein ${ }^{11}$ reportou a ausência de efeitos adversos, Shyu e $\mathrm{col}^{12}$ exploraram o potencial terapêutico do G-CSF em A.V.C. agudo isquêmico da artéria cerebral média, utilizandose dos seguintes critérios: diagnóstico de AVC através de ressonância magnética à admissão hospitalar; déficit neurológico avaliado pela escala de Stroke do NIH (NIHSS) e selecionados quando entre 9 e 20; período máximo de inclusão de 7 dias após início do AVC. Num período de 12 meses póstratamento, os pacientes que receberam G-CSF (n=7) apresentaram melhora muito mais expressiva na função neurológica do que os pacientes controle $(n=3)$ em todas as escalas clínicas utilizadas, incluindo NIHSS, escala de Stroke européia, e índice Barthel.

Relatamos neste comunicado nossa experiência com que talvez tenha sido o primeiro paciente brasileiro a fazer uso de G-CSF especificamente para uma patologia intracerebral, a qual se deu logo após a publicação de Shyu. ${ }^{12}$

Apresentamos o relato de caso de paciente de 74 anos de idade com quadro de hemorragia subaracnóidea que evoluiu para isquemia cerebral, com vaso espasmo em território das artérias cerebrais médias e posteriores bilateralmente e sistema vértebro-basilar (isquemia global). A angiografia cerebral dos vasos não mostrou origem do episódio de sangramento. A avaliação neurológica caracterizou um quadro grave de isquemia global escore GCS 3 e NIHSS 25.

Foram realizadas as medidas terapêuticas usuais - manutenção de pressão de perfusão cerebral, medidas antivasoespasmo, controle hidroeletrolítico com hipermagnesemia induzida, e terapia de suporte hemodinâmico e ventilatório gerais. Foi administrado o G-CSF na dose de $15 \mathrm{ug} /$ $\mathrm{kg} / \mathrm{dia} / \mathrm{sc}$ durante cinco dias consecutivos, a partir do $11^{\circ}$ dia da instalação da isquemia global. Foram realizados hemogramas diários e uroanálises freqüentes durante o período de internação na UTI (44 dias). Foram realizadas tomografias computadorizadas seqüenciais (anterior à administração de G-CSF, trinta e sessenta dias após) e repetida angiografia de quatro vasos (oito dias após início de G-CSF). Foi realizado SPECT sessenta dias após início G-CSF.

Observamos as alterações nos exames laboratoriais de rotina, bem como as alterações clínicas e radiológicas num período de follow-up de três meses.

Os leucócitos eram de $11.550 / \mathrm{mm}^{3}$ no dia imediatamente anterior ao G-CSF. Após o uso do G-CSF, os leucócitos se 